\title{
RESPUESTA DEL CULTIVO DE ARVEJA (Pisum sativum L.) A LA APLICACIÓN DE NÍQUEL
}

\section{PEA CROP (Pisum sativum L.) RESPONSETO THE NICKEL APPLICATION}

\author{
Miguel Ángel López Murcia1 \\ ${ }_{1}^{1}$ Docente investigador. Facultad de Ingeniería Agronómica. Universidad de Ciencias Aplicadas y Ambientales U.D.C.A. \\ milopez@udca.edu.co.
}

Rev. U.D.C.A Act. \& Div. Cient. 15(2): 357 - 362, 2012

\section{RESUMEN}

El níquel (Ni), se considera el último de los micronutrientes esenciales para las plantas superiores, debido a su participación en la enzima ureasa; sin embargo, a la fecha son pocas las investigaciones orientadas a conocer su influencia en el crecimiento y en la producción de los cultivos. Con el fin de determinar la respuesta de la arveja a la aplicación de $\mathrm{Ni}$, se realizó el montaje de un experimento en la Universidad de Ciencias Ambientales y Aplicadas U.D.C.A, donde se evaluaron, como tratamientos, cuatro dosis de Ni: 0,0; 2,0; 4,0 y 6,0 $\mathrm{mg} \mathrm{L}^{-1}$ de Ni. La investigación, se adelantó en el marco de un diseño en bloques completos al azar. Las plantas fueron sembradas en materas con arena lavada de río, como sustrato. Los resultados demuestran que la aplicación de 2,0 mg L-1 Ni promueve una mayor producción de masa seca total, así como un mayor número de vainas por planta y un mayor número de granos por vaina; no obstante, estos resultados no son estadísticamente significativos. Las concentraciones foliares de Ni variaron cercana a 0,0, en el testigo y $13,73 \mathrm{mg} \mathrm{kg}^{-1}$, en el tratamiento $6,0 \mathrm{mg} \mathrm{L}^{-1} \mathrm{Ni}$. Las concentraciones cercanas a 5,0 $\mathrm{mg} \mathrm{kg}^{-1}$ estuvieron asociadas al mayor crecimiento en las plantas de arveja.

Palabras clave: Crecimiento, producción, dosis, concentración, foliar.

\section{SUMMARY}

Nickel (Ni) is considered the last of the essential micronutrient for higher plants because of its participation in the urease enzyme. However, to date there is little research regarding its influence on growth and crop production. With the aim to determine the response of pea plants to the application of $\mathrm{Ni}$, a test was performed in the installation at the University of Applied and Environmental Sciences U.D.C.A, were four treatments were analyzed, being the doses of Ni: 0.0, 2.0;
4.0 and $6.0 \mathrm{mg} \mathrm{L}^{-1} \mathrm{Ni}$. The research was conducted in a randomized complete block design. The plants were seeded in pots containing washed river sand as a substrate. The results showed that the application of $2.0 \mathrm{mg} \mathrm{L}^{-1}$ promoted increased production of total dry mass, represented by the number of pods per plant and the number of grains per pod. However, these results were not statistically significant. The foliar concentrations of $\mathrm{Ni}$ ranged from close to 0.0 in the control and $13.73 \mathrm{mg} \mathrm{kg}^{-1}$ in the treatment $6.0 \mathrm{mg} \mathrm{L}^{-1}$, concentrations near $5.0 \mathrm{mg} \mathrm{kg}^{-1}$ were associated with higher growth in pea plants.

Key words: Growth, production, dose, concentration, leaf.

\section{INTRODUCCIÓN}

El cultivo de arveja en Colombia ha sido, por varios años, el regulador de la economía de pequeños y de medianos productores de zonas andinas, con áreas de producción, concentradas en los departamentos de Cundinamarca, de Boyacá, de Nariño y del Tolima (Buitrago et al. 2006). Fenalce (2010) reporta un área cosechada para 2008 igual a 26.826 hectáreas, con una producción calculada de $80.000 \mathrm{t}$ de vaina, para consumo fresco y $1.200 \mathrm{t}$ de arveja seca, para semilla.

El conocimiento acerca del papel del níquel (Ni) en la nutrición, en la fisiología y en el metabolismo de la mayoría de especies cultivadas en la actualidad es limitado (Bai et al. 2006) y la influencia de este elemento en el crecimiento, en al rendimiento y en la concentración foliar del cultivo de arveja, hasta la fecha, es poco conocida.

La evidencia de esencialidad del Ni para las plantas superiores no es un tema reciente, mas aún, se remonta a la década de los 70, del siglo XX, cuando ya un grupo de investigadores planteaban la posible función del níquel en el metabolismo 
del nitrógeno, a través de su participación en la estructura de la enzima ureasa (Dixon et al. 1975).

El níquel, como elemento, esta químicamente relacionado con el hierro y con el cobalto. Su estado de oxidación en los sistemas biológicos es $\mathrm{Ni}^{2+}$, aunque también puede existir como $\mathrm{Ni}^{+}$y Ni ${ }^{3+}$ (Marschner, 1995). El níquel es constituyente funcional de siete enzimas, seis, de las cuales, se presentan en bacterias y en animales, mientras que sólo una, ureasa (urea amidohidrolasa, EC 3.5.1.5), se manifiesta en plantas (Brown, 2006). La participación constitutiva del Ni en la estructura de la ureasa fue documentada, por primera vez, por Dixon et al. (1975), luego de su aislamiento y descripción a partir de Canavalia ensiformis L.

La ureasa es una metaloenzima ubicua (presente en todas partes) (Malavolta \& Moraes, 2007), conformada por seis subunidades esféricas idénticas, cada una de ellas con dos átomos de níquel (Dixon et al. 1980; Hirai et al. 1993). Aunque se considera que el níquel no es requerido para la síntesis de la ureasa, este elemento es un componente metálico esencial en la estructura y en la función catalítica de esta enzima (Hirai et al. 1993; Marschner, 1995).

La deficiencia de níquel en leguminosas y en otras dicotiledóneas ocasiona una disminución en la actividad de la enzima ureasa, condición que provoca la acumulación de niveles tóxicos de urea y se manifiesta como una necrosis en la punta de las hojas (Eskew et al. 1983; Walker et al. 1985; Malavolta \& Moraes, 2007). En soya, niveles bajos de Ni en el suelo pueden disminuir la nodulación y el rendimiento en semilla, fenómeno que se explica por la participación del $\mathrm{Ni}$ en la enzima hidrogenasa de las bacterias nitrificantes (Brown, 2006).

En Colombia, Almanza et al. (2009) evaluaron la respuesta de Cucurbita ficifolia a la aplicación de dosis crecientes de Ni. Los resultados indican que todas las dosis evaluadas $(1,0$, 2,5 y 5,0 $\mathrm{mg} \mathrm{L}^{-1} \mathrm{Ni}$ ) ocasionan algún nivel de toxicidad; sin embargo, la dosis de 5,0 $\mathrm{mg} \mathrm{L}^{-1}$ de Ni provocó la abscisión total de las flores. Los autores reportan una relación directamente proporcional entre la dosis de Ni aplicada y la cantidad de ureasa presente en las semillas.

El nivel critico foliar de Ni varía según la especie, pero, en general, para especies no acumuladoras, se considera un rango adecuado entre 1 y $10 \mathrm{mg} \mathrm{kg}^{-1}$, con base a materia seca (Marschner, 1995) y en valores superiores a $25 \mathrm{mg} \mathrm{kg}^{-1}$, provocan toxicidad en especies no acumuladoras (Malavolta \& Moraes, 2007), evidente a través de distorsiones en el crecimiento del sistema radical y en los brotes foliares (Brown, 2006). Especies hiperacumuladoras pueden llegar a presentar concentraciones de Ni del orden del 1 al 5\%, en base a materia seca (Brown, 2006).
El objetivo de esta investigación fue conocer la influencia de la aplicación de níquel en el crecimiento, componentes del rendimiento y concentración foliar de nutrientes en plantas de arveja (Pisum sativum L.).

\section{MATERIALES Y MÉTODOS}

La investigación, se desarrolló en las instalaciones de la Universidad de Ciencias Aplicadas y Ambientales U.D.C.A, ubicada a 4०47'57" Ny 74०02'40”, a 2560 msnm, temperatura promedio de $14,5 \circ \mathrm{C}$ y $860 \mathrm{~mm}$ de precipitación media anual con régimen bimodal, durante un ciclo del cultivo de arveja.

Se sembraron semillas certificadas de arveja variedad Santa Isabel, en materas plásticas, con capacidad de seis litros; se utilizó como sustrato arena de río lavada. Las plantas crecieron en condiciones de campo abierto y con manejo agronómico tradicional para manejo de plagas, de enfermedades y de prácticas culturales, de acuerdo a Buitrago et al. (2006). Para el manejo de la fertilización y la nutrición del cultivo, se empleó, en todos los tratamientos, la solución completa de Hoagland (Epstein, 2005), con una frecuencia de aplicación de tres veces por semana, a una dosis de $150 \mathrm{cc} /$ matera, en cada aplicación.

La investigación, se enmarcó en un diseño experimental de bloques completos al azar, con cuatro tratamientos y tres réplicas o bloques por tratamiento, para un total de 12 unidades experimentales, conformada, cada una, por 18 plantas de arveja. A los 30 días de emergencia, se sortearon los tratamientos y se aplicó a cada planta, de acuerdo al

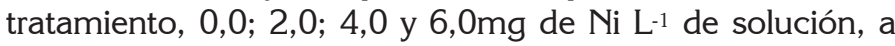
una dosis de $200 \mathrm{cc} /$ planta. Como fuente de níquel, se usó $\mathrm{NiSO}_{4} \cdot 7 \mathrm{H}_{2} \mathrm{O}$ grado comercial del $27,4 \%$ de $\mathrm{Ni}$. En este estudio, las dosis de níquel se aplicaron una sola vez durante el ciclo y constituyeron los tratamientos.

Un muestreo destructivo, inmediatamente antes de la aplicación de los tratamientos, fue adelantado, con el fin de determinar variables iniciales de peso seco de raíz, tallo, hojas, total y área foliar. Se ensayó sobre una planta por unidad experimental y los datos obtenidos, a partir del muestreo se analizaron estadísticamente, con el propósito de descartar diferencias iniciales entre tratamientos.

Posterior a la aplicación de la solución con níquel, se realizaron muestreos de una planta por unidad experimental, con el objetivo de determinar en laboratorio masa seca por órganos y total y, de esta manera, monitorear el efecto del níquel en el crecimiento de la arveja; la frecuencia de dichos muestreos fue quincenal. Los valores de masa seca obtenidos a partir de estos muestreos, se emplearon para determinar, posteriormente, los índices y las curvas de crecimiento, de acuerdo a cada tratamiento. 


\section{RESULTADOS Y DISCUSIÓN}

En la figura 1, se presenta la dinámica de ganancia y de acumulación de masa seca por plantas de arveja, sometidas a diferentes dosis de Níquel (Ni). Esta figura muestra el crecimiento de tipo sigmoidal o logístico presente en todos los tratamientos; sin embargo, sobresale el tratamiento 2,0 mg $\mathrm{L}^{-1}$, ya que promueve, en mayor proporción, la acumulación de masa seca, efecto que se empieza a evidenciar de manera marcada, a los 80 días, después de emergencia (DDE).

En contraste, el tratamiento $6,0 \mathrm{mg} \mathrm{L}^{-1}$ presenta una efecto desestimulante en la producción de masa seca, respuesta que, en principio, se puede atribuir a una dosis excesiva de $\mathrm{Ni}$, la cual, puede ocasionar síntomas leves de fitotoxicidad al interior de la planta y, por consiguiente, una disminución en la producción de masa seca (Epstein, 2005), respecto al testigo $0,0 \mathrm{mg} \mathrm{L}^{-1}$.

El aumento en la masa seca, como respuesta de la aplicación de $\mathrm{Ni}$, es un comportamiento ya antes reportado en otras especies, como Rosa de Jamaica (Hibiscus sabdariffa) (Eman et al. 2007), tomate (Lycopersicum sculentum L.) (Gad et al. 2007) y plántulas de Umbu (Spondias tuberosa Arr. Cam) (Caires et al. 2007).

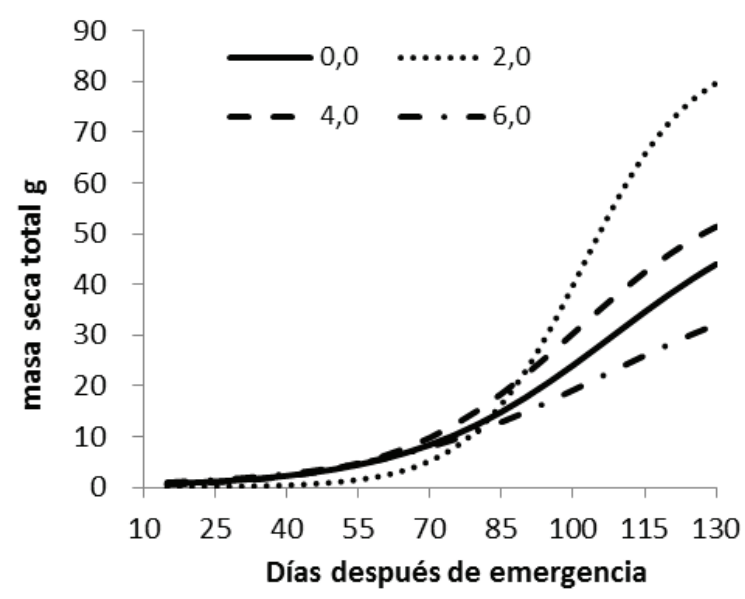

La figura 1 también presenta la dinámica de crecimiento del área foliar de plantas de arveja tratadas con diferentes dosis de Ni. Para este caso y al igual que para la masa seca, la aplicación de 2,0 $\mathrm{mg} \mathrm{L}^{-1}$ promueve un incremento importante en el área foliar de las plantas de arveja, con valores que superan en más de $800 \mathrm{~cm}^{2}$ el área foliar de los demás tratamientos, incluyendo el testigo.

La estimulación en la producción de área foliar ejercida por la aplicación de 2,0 $\mathrm{mg} \mathrm{L}^{-1}$, se podría relacionar de manera preliminar con un efecto positivo ejercido por el Ni sobre el metabolismo del nitrógeno y, en específico, sobre la metabolización de la urea (Marschner, 1995; Azcon-Bieto \& Talón, 2000; Epstein, 2005), fenómeno que favorecería la metabolización y la conversión del $\mathrm{N}$-ureico a N-orgánico o aminoácidos.

En la figura 2, se detalla el efecto de la aplicación de dosis crecientes de $\mathrm{Ni}$ en los índices de crecimiento: tasa de crecimiento relativo (TRC), tasa absoluta de crecimiento (TAC), tasa de asimilación neta (TAN) y tasa de crecimiento del cultivo (TCC) de plantas de arveja. Sobresale las elevadas TRC obtenidas en el tratamiento 2,0 $\mathrm{mg} \mathrm{L}^{-1}$ durante los primeros 70-75 DDE, comportamiento que indica una elevada habilidad por parte de las plantas tratadas con

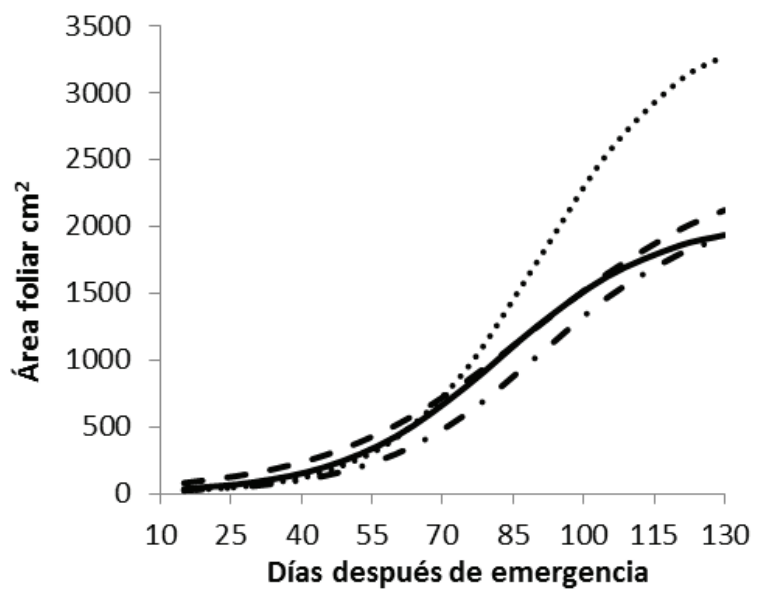

Figura 1. Dinámica de producción de masa seca y área foliar en plantas de arveja tratadas con diferentes dosis de níquel.

esta dosis de $\mathrm{Ni}$, para producir nueva biomasa, a partir de una biomasa inicial, en un intervalo de tiempo. Así, estas elevadas TRC son las que permiten explicar los mayores niveles de producción de masa seca, por parte de las plantas del tratamiento $2,0 \mathrm{mg} \mathrm{L}^{-1}$.

Una mayor producción de masa seca en un mismo lapso de tiempo, como consecuencia de la adición de $\mathrm{Ni}$, puede resultar en un mayor rendimiento por unidad de área, debido a que, posteriormente, una proporción de esta materia seca debe ser distribuida hacia las vainas u órganos de interés agronómico.

Con respecto a los demás tratamientos, se observa un comportamiento similar entre el testigo $0,0 \mathrm{mg} \mathrm{L}^{-1} \mathrm{y}$ para la dosis máxima evaluada $6,0 \mathrm{mg} \mathrm{L}^{-1}$. Así, la adición de $\mathrm{Ni}$ a dosis de $6,0 \mathrm{mg} \mathrm{L}^{-1}$ no favorece la nueva producción de biomasa en plantas de arveja con respecto a plantas con ausencia de este elemento. 
A partir de la figura 2, se establece que para todos los tratamientos, la época en la que ganan mayor masa seca por unidad de tiempo se encuentra entre los 95 y 105 DDE, resultados que permiten inferir que este tiempo es particularmente critica para el manejo del cultivo, si se considera que cualquier condicion adversa que afecte la fisiologia o el estado general de la planta ocurrida en este lapso va a disminuir de manera fuerte la produccion de masa seca de la planta y, por ende, su rendimiento potencial.

La tasa de asimilación neta TAN es una medida de la eficiencia fotosintética promedio de las plantas y mide la habilidad del vegetal para producir masa seca por cada unidad de área foliar, que capta luz en un determinada unidad de tiempo (Hunt, 1978). En la figura 2, se observa el efecto de adición de diferentes dosis de Ni sobre la TAN de plantas de arveja. Al respecto, se aprecia que los tratamientos 0,0 y $6,0 \mathrm{mg} \mathrm{L}^{-1}$ presentan un comportamiento descendente a lo largo del tiempo, con altos niveles de producción de biomasa por unidad de área foliar al inicio del cultivo, que van descendiendo, progresivamente, a medida que avanza el tiempo. Este descenso, según la literatura, se atribuye a la generación de autosombreamiento entre plantas (Hunt, 1978).
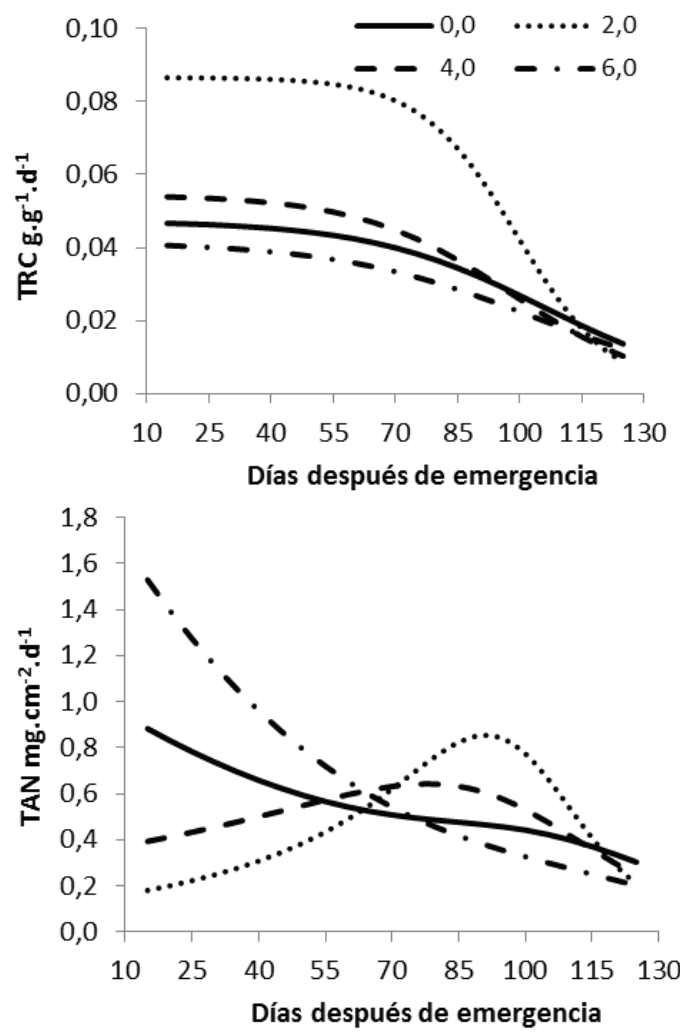

Por último, la figura 2 muestra la respuesta de la tasa de crecimiento del cultivo TCC en arveja a la adición de diferentes dosis de Ni.

Al igual que en las otros índices de crecimiento, la aplicación de Ni en dosis de 2,0 $\mathrm{mg} \mathrm{L}^{-1}$ estimula la producción de masa seca, por una comunidad de plantas de arveja. Sobresale los elevados valores de TCC, reportados por todos los tratamientos entre los 95-105 DDE, época que, como ya se había mencionado, es crucial en la definición de la máxima producción y acumulación de biomasa por planta.

En contraste, la adición de $6,0 \mathrm{mg} \mathrm{L}^{-1}$ ejerce un efecto desestimulante en la producción de biomasa por la comunidad de plantas de arveja en un lapso de tiempo definido. De esta manera y según los resultados aquí presentados, dosis superiores a $2,0 \mathrm{mg} \mathrm{L}^{-1}$ no suponen una ventaja comparativa respecto a la no adición de $\mathrm{Ni}$, en el cultivo de arveja (Figura 1).

En la tabla 1, se presenta el efecto de la aplicación de $\mathrm{Ni}$ en los componentes del rendimiento del cultivo: número de vainas por planta (NVP), peso fresco de la vaina (PFV), número de granos por vaina (NGV) y peso fresco de cien
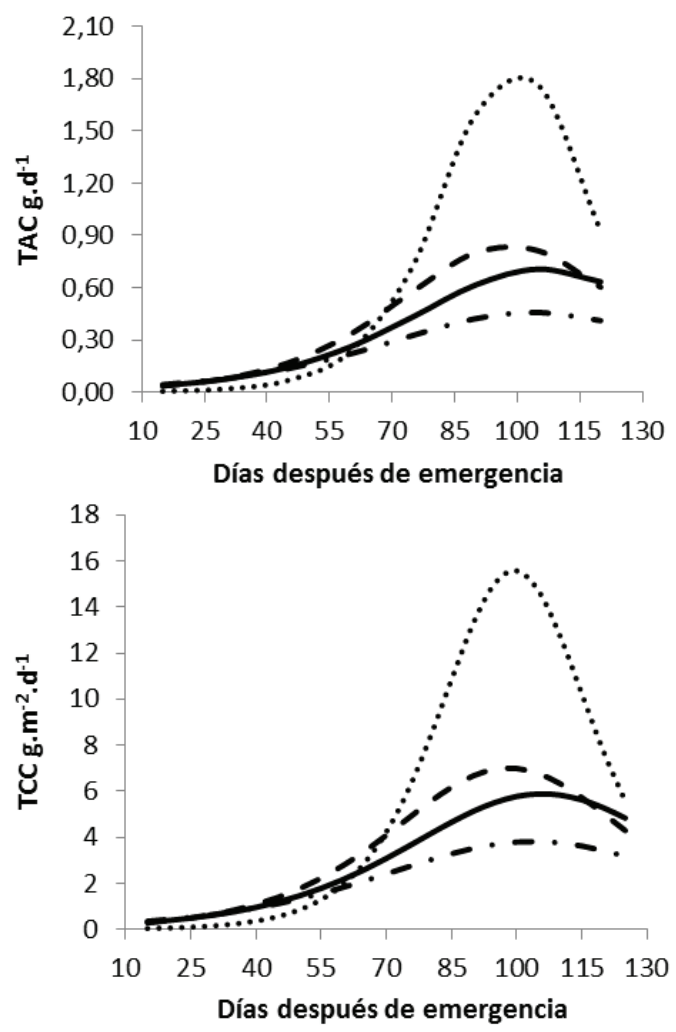

Figura 2. Respuesta de los indices de crecimiento tasa relativa de crecimiento, tasa absoluta de crecimiento, tasa de asimilacion neta y tasa de crecimiento del cultivo de plantas de arveja a la aplicación de diferentes dosis de níquel. 
granos (PCG). Sobresale la respuesta positiva ejercida por la aplicación de 2,0 $\mathrm{mg} \mathrm{L}^{-1}$ de $\mathrm{Ni}$ en el número de vainas por planta y el número de granos por vaina, con incrementos porcentuales respecto al testigo sin aplicación de 52,2 y 13,4 , respectivamente; sin embargo, estos incrementos no son estadísticamente significativos (Tabla 1).

El número de vainas por planta es una variable correlacionada de manera positiva con el rendimiento y además una de las características deseables en los procesos de mejora genética (Pacheco et al. 2010). Según González \& Ligarreto (2006), el rendimiento en arveja voluble depende de la compensación de sus componentes, siendo el número de vainas el de mayor incidencia.

Con respecto a las variables peso fresco de la vaina (PFV) y peso fresco de cien granos (PCG), no se presentan diferencias estadísticas significativas (Tabla 1). No obstante, el PCG del tratamiento $2,0 \mathrm{mg} \mathrm{L}^{-1}$ de $\mathrm{Ni}$ es el menor de todos los tratamientos evaluados, comportamiento que seguramente es el resultado de un proceso de autorregulación de los componentes del rendimiento del cultivo y, por tanto, una consecuencia visible del incremento de otros componentes, como el NVP y el NGV.
En la tabla 2, se presentan las concentraciones foliares con base a masa seca de cada tratamiento. Los resultados indican que el mayor crecimiento y producción de vainas en las plantas de arveja evaluadas en esta investigación, se encuentra asociado a concentraciones foliares iguales a $4,41 \mathrm{mg} \mathrm{kg}^{-1}$, valor coherente con los niveles planteados como adecuados para la mayoría de especies vegetales, según Marschner (1995).

De acuerdo a los resultados de la tabla 2, también se plantea que concentraciones de Ni iguales a $8,70 \mathrm{mg} \mathrm{kg}^{-1}$ no reportan una ventaja comparativa respecto a la no adición de Ni en las variables de crecimiento y de producción evaluadas. Entre tanto, la aplicación de 6,0 $\mathrm{mg} \mathrm{L}^{-1}$ de Ni se asocia con concentraciones foliares del elemento iguales a $13,73 \mathrm{mg}$ $\mathrm{kg}^{-1}$, niveles que, de acuerdo a los resultados de esta investigación, se asocian con depresión en el crecimiento y en la producción de la arveja y, probablemente, con inicios de toxicidad.

A partir de los resultados de la investigación, se puede concluir que la aplicación de $\mathrm{Ni}$ en dosis de 2,0 $\mathrm{mg} \mathrm{L}^{-1}$ promueve un incremento en la producción de masa seca y número de vainas por planta en arveja. Estos aumentos

Tabla 1. Efecto de la aplicación de níquel en los componentes del rendimiento del cultivo de arveja (nvp: número de vainas por planta; pfv: peso fresco de la vaina; ngv: número de granos por vaina; pcg: peso fresco de cien granos; de: desviación estándar).

\begin{tabular}{|c|c|c|c|c|c|c|c|c|}
\hline $\begin{array}{c}\text { Dosis de Ni } \\
\mathrm{mg} \mathrm{L}^{-1}\end{array}$ & NVP & DE & PFV $(\mathrm{g})$ & DE & NGV & DE & PCG (g) & DE \\
\hline 0,0 & 29,67 & 12,89 & 5,29 & 0,49 & 5,16 & 0,60 & 36,05 & 5,61 \\
\hline 2,0 & 45,17 & 27,33 & 5,79 & 1,11 & 5,85 & 0,45 & 34,59 & 5,65 \\
\hline 4,0 & 30,67 & 18,18 & 5,52 & 0,55 & 5,19 & 0,57 & 37,61 & 4,02 \\
\hline 6,0 & 21,17 & 11,41 & 5,26 & 0,94 & 4,96 & 1,43 & 38,76 & 8,80 \\
\hline $\mathrm{P}^{*} \mathrm{~F}^{*}$ & 0,236 & & 0,665 & & 0,282 & & 0,617 & \\
\hline
\end{tabular}

* valor de probabilidad obtenida a partir del analisis de varianza.

Tabla 2. Contenidos de nutrientes en hojas de plantas de arveja tratadas con diferentes dosis de níquel.

\begin{tabular}{|c|c|c|c|c|c|c|c|c|c|c|c|c|c|}
\hline $\begin{array}{c}\text { Dosis de } \\
\text { Ni mg L-1 }\end{array}$ & N & P & K & Ca & Mg & Na & S & Cu & Fe & Zn & Mn & B & Ni* \\
\hline 0,0 & 2,59 & 0,18 & 1,10 & 0,93 & 0,21 & 0,02 & 0,21 & 4,50 & 91,50 & 55,50 & 22,30 & 22,60 & 0,0007 \\
\hline 2,0 & 2,64 & 0,20 & 1,15 & 1,12 & 0,25 & 0,01 & 0,24 & 5,00 & 64,00 & 61,50 & 36,10 & 32,10 & 4,41 \\
\hline 4,0 & 2,07 & 0,17 & 0,95 & 1,67 & 0,21 & 0,01 & 0,20 & 3,70 & 76,50 & 70,90 & 44,40 & 31,10 & 8,70 \\
\hline 6,0 & 2,52 & 0,19 & 0,82 & 0,86 & 0,22 & 0,01 & 0,19 & 4,70 & 82,70 & 56,60 & 25,60 & 18,50 & 13,73 \\
\hline
\end{tabular}

*Extraído a partir de digestión seca y cuantificado por absorción atómica. 
aunque son biológicamente importantes con respecto al testigo sin aplicación, no son estadísticamente significativos.

Agradecimiento: A la Universidad de Ciencias Aplicadas y Ambientales U.D.C.A, por la financiación de la investigación. Conflictos de intereses: El manuscrito fue preparado y revisado por el autor, quien declara que no existe ningún conflicto de intereses que ponga en riesgo la validez de los resultados presentados.

\section{BIBLIOGRAFÍA}

1. ALMANZA, P.J.; ROJAS, H.A.; BORDA, G DEL C.; GALINDO, A.R.; GALINDO, D.R. 2009. Aplicación foliar de níquel en Cucurbita ficifolia Bouché para producción de ureasa (EC 3.5.1.5) cristalina. Agr. Col. 27(1):33-40.

2. AZCON-BIETO, J.; TALÓN, M. 2000. Fundamentos de Fisiología Vegetal. Mc Graw Hill Interamericana. Barcelona. p.83-97.

3. BAI, C.; REILLY, C.; WOOD, B.W. 2006. Nickel deficiency disrupts metabolism of ureides, amino acids, and organicaAcids of young pecan foliage. Plant Physiol. 140:433-443.

4. BROWN, P.H. 2006. Nickel. In: Barker, A.V.; Pilbeam, D.J. (eds.). Handbook of Plant Nutrition. CRC Press: Taylor \& Francis Group, Boca Raton. p.329-350.

5. BUITRAGO, J.Y.; DUARTE, C.J.; SARMIENTO, A. 2006. El cultivo de la arveja en Colombia. Federación Nacional de Cultivadores de Cereales y LeguminosasFENALCE y Fondo Nacional Cerealista. Ed. Produmedios. Bogotá. Colombia. 83p.

6. CAIRES, O.S.; DE OLIVEIRA, E.V.; DE CARVALHO, J.G.; FONSECA, C.R. 2007. Adição de níquel na solução nutritiva para o cultivo de mudas de umbuzeiro. Rev. Bras. Cienc. Solo. 31:485-490.

7. DIXON, N.E.; GAZZOLA, C.; BLAKELEY, R.L.; ZERNER, R. 1975. Jack bean urease. A metalloenzyme. A simple biological role for nickel. J. Am. Chem. Soc. 97:4131-4133.

8. DIXON, N.E.; BLAKELEY, R.L.; ZERNER, R.. 1980. Jack-Bean urease III. The involvement of active site $\mathrm{Ni}$ ion in inhibition by $\mathrm{b}$ mercaptoethanol and phosphoramidate, and fluoride. Can. J. Biochem. 58:481-488.

9. EMAN, A.; GAD, N.; BADRAN, N. 2007. Effect of cobalt and nickel on plant growth, yield and flavonoids content of Hibiscus sabdariffa L. Australian J. Basic Appl. Sci. 1(2):73-78.

10. EPSTEIN, E.; BLOOM, A. 2005. Mineral nutrition of plant: principles and perspectives. Ed. Sinauer Associates, inc. Publishers. Sunderland, Massachusetts. 380p.

11. ESKEW, D.L.; WELCH, R.M.; CARY, E.E. 1983. Nickel an essential micronutrient for legumes and possibly all higher plants. Science. 222:621-623.

12. FENALCE. 2010. El cultivo de la arveja, historia e importancia. El Cerealista. 93:32-35.

13. GAD, N.; EL-SHERIF, M.H.; EL-GEREENDLY, N.H.M. 2007. Influence of nickel on some physiological aspects of tomato plants. Australian J. Basic Appl. Sci 1(3):286-293.

14. GONZÁLEZ, F.; LIGARRETO, G.A. 2006. Rendimiento de ocho genotipos promisorios de arveja arbustiva (Pisum sativum L.) bajo sistema de agricultura protegida. Fitotecn. Col. 6(2):5 2-61.

15. HIRAI, M.; KAWAI-HIRAI, R.; HIRAI, T.; UEKI, T. 1993. Structural change of jack bean urease induced by addition of surfactants studied with synchrotronradiation small-angle X-ray scattering. Eur. J. Biochem. 215:55-61.

16. HUNT, R. 1978. Plant growth analysis. Edward Arnold Publishers. London. p.8-38.

17. MALAVOLTA, E.; MORAES, M.F. 2007. Nickel - from toxic to essential nutrient. Better Crops With Plant Food. 91(3):26-27.

18. MARSCHNER, H. 1995. Mineral nutrition of higher plants. $2^{\text {nd }}$ ed. London, Academic Press. 889p.

19. PACHECO, C.A.; VERGARA, M.C.; LIGARRETO, G.A. 2010. Clasificación de 42 líneas mejoradas de Arveja (Pisum sativum L.) por caracteres morfológicos y comportamiento agronómico. Rev. Fac. Nal. Agr. Medellín. 63(2):5543-5553.

20. WALKER, CD.; GRAHAM, RD.; MADISON, JT.; CARY, E.E.; WELCH, RM. 1985. Effects of nickel deficiency on some nitrogen metabolites in cowpeas (Vigna unguiculata L. Walp.). Plant Physiol 79:474-479.

Recibido: Marzo 15 de 2012

Aceptado: Agosto 27 de 2012 